\title{
The Correlations Between Concentrations of Myeloperoxidase, Serum Amyloid-A Protein and Scretory Phospolipase A-2 with Proinflammatory HDL in Healthy Male Person
}

\author{
Marita Kaniawati ${ }^{1,3}$, Andi Wijaya ${ }^{1,3}$, and Anwar Santoso ${ }^{2}$
}

B

ACKGROUND: Low-HDL cholesterol is a risk factor of CAD. Although levels of HDL$\mathrm{C}$ are within normal limit in some patients, they suffer CAD. These normal HDL-C levels might become pro-inflammatoric. This study is to measure the correlations between myeloperoxidase (MPO), serum amyloid-A (SAA) protein, and secretoryphospholipase-A2 (sPLA2) with inflammatory status of HDL-C.

METHODS: This was a cross-sectional study recruited 49 subjects with high HDL-C (> $40 \mathrm{mg} /$ $\mathrm{dL})$ and 31 subjects with low HDL-C $(<40 \mathrm{mg} / \mathrm{dL})$. HDL-C was determined into antiinflammatory and proinflammatory based on levels of Apo A-1 and hs-CRP. Concentrations of MPO, SAA and s-PLA2 were measured by ELISA method. Levels of Apo A1 was determined by immunoturbidimetric method. Multiple logistic regression analysis was done using inflammatory status of HDL-C as dependent variables and levels of MPO, SAA, sPLA2, ages, total cholesterol and triglycerides as independent variables.

RESULTS: Patient's age was $43.4+8.3$ year, HDL-C was $43.1+9.5 \mathrm{mg} / \mathrm{dL}$, Apo A-1 was $128.3+21.5 \mathrm{mg} /$ $\mathrm{dL}$, hs-CRP was $1.92+3.0 \mathrm{mg} / \mathrm{dL}$. Concentrations of MPO, SAA and sPLA2 successively were $63.2+16.9$ $\mathrm{ng} / \mathrm{mL}, 7015.6+5021.1 \mathrm{ng} / \mathrm{mL}$ and $1340.2+406.3 \mathrm{pg} /$ $\mathrm{mL}$. Multiple logistic regression analysis showed that SAA is an independent predictor of pro-inflammatory status of HDL-C in high HDL-C group with prevalence ratio of $11.74(95 \% \mathrm{CI}: 2.51-54.84 ; \mathrm{P}=0.002)$. In contrast, $\mathrm{MPO}$ and sPLA2 were not independent predictor with PR of $1.26(95 \%$ CI : $0.30-5.23 ; \mathrm{P}=0.75)$ and of 0.94 (95\% CI : $0.23-3.91 ; \mathrm{P}=0.93)$.
CONCLUSIONS: SAA is an independent predictor of pro-inflammatory HDL-C even in subjects with high HDL-C.

KEYWORDS: Atherosclerosis, Apo A-I, serum amyloid A protein, secretory phospholipase A2, myeloperoxidase

Not all HDL (High Density Lipoprotein) protect against plaque formation in the arterial walls. This is because in some people HDL was dysfunction, or malformed, and unable to do its job properly. The fact that sometimes HDL doesn't do its job properly can explain why some people who have high HDL levels still get heart attacks and suffer from other cardiovascular disease.

Some researchers found that HDL becomes dysfunction when an enzyme from white blood cells, called myeloperoxidase (MPO), binds to Apo A-1 and modifies it. Once modified, Apo A-1 blocks HDL's ability to perform its normal physiological function.

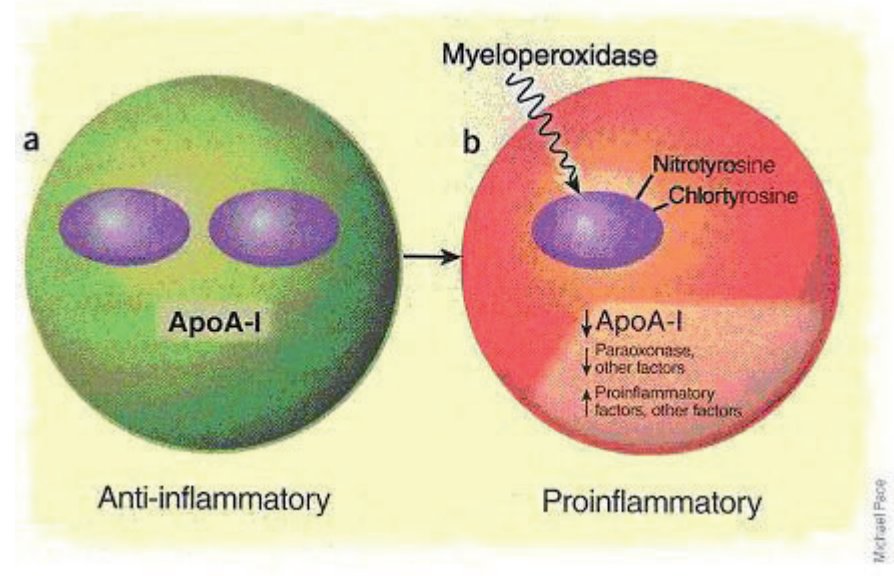

Figure 1. Converting the HDL from anti-inflammatory to proinflammatory (Adapted from Fogelman AM, 2004) 
SAA (Serum Amyloid A) is transported in the circulation primarily in association with HDL. SAA levels increase rapidly in the blood of humans suffering from acute and chronic inflammation. SAA has been proved can replace Apo A-1 in HDL in vitro. Because SAA binds to proteoglycans, SAA-containing HDL could bind to extracellular vascular proteoglycans, which would favor the retention and modification of HDL by the vascular matrix. Retention could prevent HDL from participating in the reverse cholesterol transport and inhibiting oxidative processes in the arterial wall. Modifications of lipid and protein components of trapped HDL might increase its interactions with macrophage scavenger receptors.

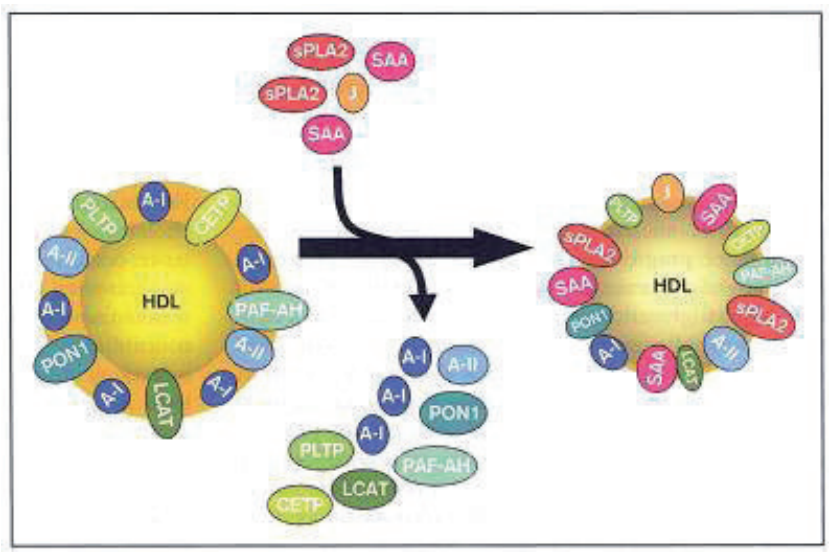

Figure 2. During the acute-phase reaction the protein and lipid composition of the HDL changes rapidly (Adapted from Rohrer, 2004)

sPLA2 (secretory Phospholipase A2) also had a role as atherosclerotic marker and mediator in human. Like SAA, it also can be bound to HDL.

High level of MPO, SAA and sPLA2 can change HDL characteristics from antiinflammatory to proinflammatory, which means can change HDL quality. If so, HDL quality actually become more important than HDL quantity.

It might also be that the strategy of increasing HDL levels per se is not enough, and some researchers believe that it is the function oh HDL that needs to be improved rather than its levels (1).

HDL quality can be determined by its lipid contents (including oxidized lipid) and apolipoprotein in HDL, particle size and electrophoretic mobility, related enzymatic activity, inflammatory/antiinflammatory properties and ability to promote cholesterol efflux (2).

A few studies to date have investigated the correlation between SAA, SPLA2 and MPO with the change of HDL characteristics from antiinflammatory to proinflammatory. According to that reason, we need to study about the correlation between SAA, sPLA2 and MPO with proatherogenic HDL. Proinflammatory HDL term was used when one of the atheroprotective HDL characteristics was disturbed. Proinflammatory HDL were determined by ApoA-I and hsCRP concentration.

\section{Patients and Methods}

Forty nine (49) healthy male person with high Cholesterol-HDL ( $\geq 40 \mathrm{mg} / \mathrm{dL}$ ), aged 33-62 years (man) were enrolled in this cross sectional study. Thirty one (31) healthy male person with low Cholesterol-HDL $(<40 \mathrm{mg} / \mathrm{dL})$ aged 30-59 years also were analysed. Participants signed informed consents to have personal data such as height, weight, smoking, serum measurements, blood pressure, waist circumference, exercises, alcohol, drugs.

\section{Assays of Biochemical Markers}

Serum Amyloid A (SAA) Protein, secretory Phospholipase A2 (sPLA2) and MPO concentrations were measured with Enzyme Linked Immuno Assay kit from Anogen, Cayman, and Calbiochem respectively. Serum were separated from whole blood after centrifugation, and immediately kept at $-20 \mathrm{oC}$ until assays. All assays were performed according to manufacturers instruction.

For each run of SAA Protein, sPLA2 and MPO, controls were included in the assays, and all results were within acceptable ranges. In our laboratory, the intraday imprecisions for all assays were between $4.2 \%-5.4 \%$.

\section{Statistical Analysis}

Statistical analyses were performed with the SPSS for Windows version 13.0 software. Univariate, bivariate and multivariate analysis were calculated. The results were narrated and explained by tables and graphics.

- $\quad$ For statistical test, we used 5\% significance. 


\section{Result s}

Patient's age was $43.4 \pm 8.3$ year, HDL-C was $43.1 \pm 9.5 \mathrm{mg} / \mathrm{dL}$, Apo A-1 was $128.3 \pm 21.5 \mathrm{mg} / \mathrm{dL}$, hs-CRP was $1.92 \pm 3.0 \mathrm{mg} / \mathrm{dL}$. Concentrations of MPO, SAA and sPLA2 successively were $63.2 \pm 16.9 \mathrm{ng} / \mathrm{mL}, 7015.6 \pm 5021.1$ $\mathrm{ng} / \mathrm{mL}$ and $1340.2 \pm 406.3 \mathrm{pg} / \mathrm{mL}$.

Table 1. Median levels of Apo A-1, SAA, sPLA2, MPO and hs-CRP

\begin{tabular}{|c|c|c|c|c|c|}
\hline & $\begin{array}{c}\text { Apo A-1 } \\
(\mathrm{mg} / \mathrm{dL})\end{array}$ & $\begin{array}{c}\mathrm{SAA} \\
(\mathrm{ng} / \mathrm{mL})\end{array}$ & $\begin{array}{c}\mathrm{sPLA} 2 \\
(\mathrm{pg} / \mathrm{mL})\end{array}$ & $\begin{array}{c}\mathrm{MPO} \\
(\mathrm{ng} / \mathrm{mL})\end{array}$ & $\begin{array}{c}\text { hs-CRP } \\
(\mathrm{mg} / \mathrm{L})\end{array}$ \\
\hline N8 & 08 & 07 & 97 & 98 & 0 \\
\hline Median & $\begin{array}{c}125,00 \\
(90-201)\end{array}$ & $\begin{array}{c}5905,00 \\
(423,2-20.410,0)\end{array}$ & $\begin{array}{c}1316,00 \\
(293-2565)\end{array}$ & $\begin{array}{c}62,30 \\
(32,7-101,8)\end{array}$ & $\begin{array}{c}1,10 \\
(0,1-22,4)\end{array}$ \\
\hline
\end{tabular}

SAA = Serum Amyloid A; SPLA2 = secretory Phospholipase A2; MPO = Myeloperoxidase; hs-CRP = high sensitivity C-Reactive Protein

HDL-C was defined as proinflammatory based on levels Apo A- $1<125 \mathrm{mg} / \mathrm{dL}$, and or hs-CRP $>1,1 \mathrm{mg} / \mathrm{L}$. From 49 subjects with high HDL-C ( $\geq 40 \mathrm{mg} / \mathrm{dL}$ ), by using that criteria we found 28 subjects with antiinflammatory properties and 21 subjects $w$ ith proinflammatory properties. Statistical analysis $w$ as made u sing independent sample $t$ test to see the different between SAA, sPLA2 and MPO.

Table 2. Level of Apo A-1, SAA, sPLA2, MPO and hs-CRP among HDL type (antiinflammatory/ proinflammatory) in high HDL-C group

\begin{tabular}{|c|c|c|c|c|}
\hline & HDL type & $\mathrm{N}$ & Mean \pm SD & $P$ \\
\hline Apo A-1 & $\begin{array}{l}\text { Antiinflammatory } \\
\text { Proinflamamatory }\end{array}$ & $\begin{array}{l}28 \\
21\end{array}$ & $\begin{array}{l}144,61 \pm 20,83 \\
132,09 \pm 15,26\end{array}$ & 0,025 \\
\hline SAA & $\begin{array}{l}\text { Antiinflammatory } \\
\text { Proinflamamatory }\end{array}$ & $\begin{array}{l}28 \\
21\end{array}$ & $\begin{array}{c}5303,42 \pm 3967,15 \\
10903,86 \pm 4546,75\end{array}$ & 0,000 \\
\hline sPLA2 & $\begin{array}{l}\text { Antiinflammatory } \\
\text { Proinflamamatory }\end{array}$ & $\begin{array}{l}28 \\
21\end{array}$ & $\begin{array}{l}1216,21 \pm 386,14 \\
1453,38 \pm 391,55\end{array}$ & 0,040 \\
\hline MPO & $\begin{array}{l}\text { Antiinflammatory } \\
\text { Proinflamamatory }\end{array}$ & $\begin{array}{l}28 \\
21\end{array}$ & $\begin{array}{l}60,20 \pm 16,80 \\
61,26 \pm 18,03\end{array}$ & 0,833 \\
\hline hs-CRP & $\begin{array}{l}\text { Antiinflammatory } \\
\text { Proinflamamatory }\end{array}$ & $\begin{array}{l}28 \\
21\end{array}$ & $\begin{array}{l}0,64 \pm 0,31 \\
3,74 \pm 5,20\end{array}$ & 0,003 \\
\hline
\end{tabular}

HDL = High Density Lipoprotein; SAA = Serum Amyloid A; sPLA2 = secretory Phospholipase A2; MPO = Myeloperoxidase; hs-CRP = high sensitivity C-Reactive Protein 
Tabel 3. Multiple logistic regresion analysis

\begin{tabular}{|l|c|c|c|c|c|}
\hline \multicolumn{1}{|c|}{ Variabel } & B & Wald & $\mathrm{p}$ & Prevalence ratio & $(95 \% \mathrm{Cl})$ \\
\hline SAA & 2,463 & 9,816 & 0,002 & 11,744 & $(2,515-54,839)$ \\
sPLA2 & $-0,061$ & 0,007 & 0,933 & 0,940 & $(0,226-3,912)$ \\
MPO & 0,235 & 0,104 & 0,747 & 1,265 & $(0,304-5,272)$ \\
age & $-0,060$ & 1,689 & 0,194 & 0,942 & $(0,861-1,031)$ \\
total chol. & $-0,009$ & 0,285 & 0,594 & 0,992 & $(0,961-1,023)$ \\
tryglyceride & 0,007 & 1,134 & 0,287 & 1,007 & $(0,994-1,020)$ \\
Constanta & 1,455 & 0,175 & 0,676 & 4,285 & - \\
\hline
\end{tabular}

SAA = Serum Amyloid A; sPLA2 = secretory Phospholipase A2; MPO = Myeloperoxidase

Multiple logistic regression analysis showed that SAA is an independent predictor of pro-inflammatory status of HDL-C in high HDL-C group with prevalence ratio of 11.74 (95\% CI : $2.51-54.84 ; \mathrm{P}=0.002)$. In contrast, $\mathrm{MPO}$ and sPLA2 were not independent predictor with PR of 1.26 (95\% CI : $0.30-5.23 ; \mathrm{P}=0.75)$ and of $0.94(95 \% \mathrm{CI}$ : $0.23-3.91 ; \mathrm{P}=0.93)$.

\section{Discussions}

Normally, HDL exert a range of potent biological activities that provide protection against atherosclerosis. These actions include a key role in mediating cholesterol efflux from plaque macrophages and foam cells, and from peripheral tissues, with transport to the liver for excretion via the process of reverse cholesterol transport; equally, anti-oxidant, anti-inflammatory, antiapoptotic, anti-thrombotic, anti-infectious and vasodilatory properties are central features of HDLmediated atheroprotection. HDL particles are highly heterogeneous in terms of their physico-chemical characteristics, structure, intravascular metabolism and biological activity.

The role of HDL in atherosclerosis is not apart from the role of Apo A-1. Apo A-1 ability to promote reverse cholesterol transport and remove seeding molecule from LDL (important in oxidized phospholipids formation, originate from LDL inflammatory) contribute to inverse relationship between HDL-cholesterol level and susceptibility to atherosclerosis (3).
HDL can bind and neutralize lipopolysaccharide, to modulate acute and chronic inflammation. Flowcytometry analysis shows that Apo A- 1 associated with HDL can inhibit monocyte activation by binding to T lymphocyte, to inhibit tumor necrosis factor (TNF)$\alpha$ and interleukin- $1 \beta$ production, essential component on immunoinflammatory diseases pathogenesis. It has been hypothesised that increasing HDL have an effect on antiinflammation action (5).

In this study, subject were divided into high HDL group (HDL $\geq 40 \mathrm{mg} / \mathrm{dL}, \mathrm{n}=49$ ) and low HDL group (HDL $<40 \mathrm{mg} / \mathrm{dL}, \mathrm{n}=31$ ). Based on median of Apo A$1(125 \mathrm{mg} / \mathrm{dL})$ and hs-CRP $(1,1 \mathrm{mg} / \mathrm{L})$ we determined HDL antiinflammatory type if the subject has Apo A-1 $\geq 125 \mathrm{mg} / \mathrm{dL}$ and/or has hs-CRP $<1,1 \mathrm{mg} / \mathrm{L}$, and HDL proinflammatory if the subject has Apo A$1<125 \mathrm{mg} / \mathrm{dL}$ and/or has hs-CRP $\geq 1,1 \mathrm{mg} / \mathrm{L}$. From 49 subjects with high HDL we found 28 subject with HDL antiinflammatory type and 21 subjects with HDL proinflammatory. From subjects with low HDL we found only 1 subject with HDL anti-inflammatory type and 30 subjects with HDL proinflammatory type. 
Table 4. The type of HDL in high HDL and low HDL groups

\begin{tabular}{|l|c|c|c|}
\hline HDL group & HDL antiinflammatory & HDL proinflammatory & Total \\
\hline $\mathrm{HDL} \geq 40 \mathrm{mg} / \mathrm{dL}$ & 28 & 21 & 49 \\
\hline $\mathrm{HDL}<40 \mathrm{mg} / \mathrm{dL}$ & 1 & 30 & 31 \\
\hline Total & 29 & 51 & 80 \\
\hline
\end{tabular}

HDL = High Density Lipoprotein

In our study from 49 subjects with $\mathrm{HDL} \geq 40$ $\mathrm{mg} / \mathrm{dL}$ there are 21 subjects $(42,86 \%)$ who have HDL proinflammatory type and 28 subjects $(57,14 \%)$ having HDL anti-inflammatory type. It means that in high HDL group, we found subject with HDL proinflammatory type. So, if we just only think about quantity of $\mathrm{HDL}$, we cannot assured of its quality. On the other hand, we found in low HDL group 30 subjects with HDL proinflammatory type $(96,77 \%)$.

From the whole population study $(\mathrm{n}=80), 51$ subjects $(63,75 \%)$ have HDL proinflammatory type and 29 subjects $(36,25 \%)$ have HDL anti-inflammatory type.

From high HDL group, that we were focusing on this study, we found 28 subjects with high SAA $(57,14 \%), 25$ subjects with high sPLA2 $(51,02 \%)$ and 22 subjects with high MPO (44,90\%). Possibly because there is big relationship with the finding of HDL proinflammatory in high HDL group. In some subjects with high HDL level (formerly thought to be protective) when accompanied by high MPO, and/or high SAA, and/or high SPLA2, the HDL function will be disturbed, that shown by low Apo A-1 and/or high hs-CRP.

From our study we found :

- there was significant positive correlation between SAA with HDL proinflammatory, with prevalence ratio $10,800, p=0,001$.

- there was weak positive correlation between sPLA2 with HDL proinflammatory, prevalence ratio $1,100, p=0,869$.

- there was weak positive correlation between MPO with HDL proinflammatory with prevalence ratio $0,865, \mathrm{p}=0,804$.

- SAA is the best independent predictor by multivariate logistic regression analysis with prevalence ratio 11,$744 ; \mathrm{p}=0,002$, followed by MPO and SPLA2.

\section{Interaction between SAA, SPLA2 and MPO in HDL proinflammatory}

Several epidemiological studies showed inverse relationship between HDL and artery coroner disease risk. Part of HDL protective effect is caused by LDL oxidative modification inhibition or by promoting cholesterol efflux from pheriferal cells. In certain condition, eg. acute phase reactant, native antioxidant properties of HDL were changed. Just the opposite from native HDL that inhibit LDL modification and monocyte adhesion to endothelial cells in artery cocultur system, acute phaseHDL will increase monocyte adhesion. In acute phase, lipoprotein composition change dramatically from non acute phase condition. In this condition, HDL showed big increasing SAA, with decreasing Apo A-1 level. HDLcontaining Apo A-1 particle consist of lecithin cholesteryl acyl transferase (LCAT), that responsible for plasma cholesterol esterification and acting as HDL ligand that has role in HDL interaction with cell. Apo A-1 substitution by SAA within acute phase can inhibit LCAT activity in HDL, repressing cholesterol efflux from pheriferal cells (6).

Recent study has evaluated HDL protective effect. HDL-enriched with SAA does not have protective effect. This study suggested that within acute phase, joining SAA into HDL can cause changing in HDL particle, and diminished protective capacity to LDL oxidation. Acute phase-HDL is not only inhibit LDL oxidation but also result in monocyte migration (6).

In our study, we found 28 person $(57,14 \%)$ who has high level of SAA in high HDL group. By using univariate logistic regression analysis we found that in high HDL group, SAA is the best independent predictor with prevalence ratio $10,800(95 \% \mathrm{CI}$ : 2,543 - 45,866), p : 0,001, on the other hand based on multivariate regression analysis shown that in high HDL group SAA is still best independent predictor 
with prevalence ratio $11,744(95 \% \mathrm{CI}: 2,515-54,839)$ p : 0,002 , followed by MPO and sPLA2 respectively. It means that serum SAA level is significantly associated and independent with proinflammatory HDL type.

This result similar with van Lenten finding. They found decreasing $73 \%$ Apo A-1 level in people with high SAA level. People with high level of HDL, if accompanied by high SAA can have proinflammatory HDL type.

Secretory Phospholipase A2 (sPLA2) is an enzyme that break acyl ester in sn-2 position in glycerophospholipid when activated. sPLA2 is extracellular enzyme with high disulphide binding, with low molecular weight (14 $\mathrm{KDa}$ ), and need $\mathrm{mM} \mathrm{Ca} 2+$ for catalysis. This enzyme has important role in arachidonic and eicosanoid formation, proinflammatory mediator. PLA2 has several biological action such as immunologic respons, inflammation, cellular proliferation, vasoconstriction and bronchioconstriction.

Circulating sPLA2 level will increase dramatically within infection and inflammation. In nonatherosclerotic artery, sPLA2 type IIA especially associated with smooth muscle cells. In atherosclerotic lesion, this enzyme associated with macrophage, necrotic lipid core and extracellular vascular proteoglican. This enzyme was bound with very high affinity (7).

Our study found 25 person (51\%) who has high sPLA2 in high HDL group. Based on univariate logistic regression analysis SPLA2 was not proven associated significantly with proinflammatory HDL type. This is not similar with former reports that mention that in person with high HDL level, if accompany by high sPLA2, his HDL will change to proinflammatory type.

There are several possibilities as a cause for these differences. First, there are some steps in the testing procedure that should be paid attention. The higher standard to achieve standard curve is $1.000 \mathrm{pg} / \mathrm{mL}$, and our results are in the range 293 to $2.696 \mathrm{pg} / \mathrm{mL}$. Mean $+\mathrm{SD}: 1340,18+406,27 \mathrm{pg} / \mathrm{mL}$. Diluting factor is very important if we want to continue this research. Second, absorbance reading also can be done several times (15, $30,60,120,240,360$ minutes). In our research, we used value only at 15 and 30 minutes reading, and we calculate the difference. Perhaps, this reading method influence the value. Third, we used sPLA2 (human Type IIa) EIA kit that measure SPLA2 concentration.
Maybe it will be better to use kit that measure sPLA2 activity, because measurement of enzyme activity generally has better correlation than measuring the concentration. Fourth, the number of research sample too small so it is not enough to prove the double hypothesis in this research. Fifth, the recent researchs usually predict the correlation between high sPLA2 with proinflammatory properties of low HDL. We want to know wheter the influence of this high sPLA2 can happen in high HDL group with proinflammatory properties. Our analysis shown that SPLA2 mean in high HDL group with proinflammatory properties higher than sPLA2 mean in low HDL group. Possibly sPLA2 can influence to change HDL properties, from anti-inflammatory to proinflammatory, but this effect need long time to occur.

Modification by MPO product, can cause Apo A-1 bind tightly to the artery. Similarly with the finding in Fogelman study that in atherosclerosis artery, we can find Apo A-1 in a big amount. In a non modified-Apo A-1, on the contrary, the normal Apo A-1 doesn't have binding site with heparin. So we can find more Apo A-1 in plasma than in artery (8). Level of hs-CRP $>3 \mathrm{mg} / \mathrm{L}$ will strengthen the prediction about inflammation and showing the risk to get cardiovascular disturbances in the future if doesn't treat earlier.

Even oxidation product by MPO can be found more abundant in circulation HDL in individu with CVD and atherosclerosis lesion, in fact significant amount also can be found in Apo A-1 from the healthy individual serum (9).

We found 22 individu (44,9\%) with high MPO in high HDL group. Based on univariate logistic regression analysis, the prevalence ratio is $0,865(95 \% \mathrm{CI}: 0,277-2,705)$ with $\mathrm{p}=0,804$. With multivariate logistic regression analysis, the prevalence ratio is $1,265(95 \% \mathrm{CI}: 0,304-5,272)$ with $\mathrm{p}=$ 0,747 . It means that MPO doesn't have significant association with proinflammatory-HDL. It is different with the previous studies which show that in individu with high HDL accompany by high MPO, the HDL become proinflammatory HDL.

There are some possibilities as the cause of this differences. First, Apo A-1 oxidation catalyzed by MPO in arterial wall or in circulation, not followed by selective deposition of modified lipoprotein in arterial wall, so Apo A-1 still can be measured in the blood. Besides, we must remember that - different from LDL that has only one Apo B on its each particle- more than one Apo A-1 can be found on each HDL particle. This 
deposition issue was still a question mark for previous researchers and has not yet been answered by existing studies. Factors that influence the modified Apo A1 deposition in the artery wall have to be found out. Second, the process of Apo A-1 modification progress slowly that it takes quite a long time to be able to alter the HDL function to become pro-inflammatory. Other studies using the case control or cohort designs are probably needed. Subjects with elevated MPO can be reexamined at another time for the HDL type, to see if there is any change to pro-inflammatory HDL. Third, to get a better understanding about the proinflammatory $\mathrm{HDL}$, we indeed need to measure not only the Apo A-1 concentration, but also the 3chlorotyrosine in HDL using mass spectrophotometry. Increased 3-chlorotyrosine in HDL indicated high level of Apo A-1 modification by MPO (10).

\section{Relationship between Study Results and Epidemiological Data}

Recently, several reports had given a number of data supporting the statement of the involvement of MPO in HDL pro-inflammatory characteristics. This is suggested to associate with the MPO ability (through its products) to modify Apo A-1. When Apo A-1 is modified by MPO, it will block the normal HDL function. There is an inverse relationship between the amount of tyrosine in apoA-I modified by MPOderived products and the ability of HDL to remove cholesterol from macrophages. Thus, the more tyrosine in apoA-I that is modified by MPO-derived products, the less effective the HDL is in removing cholesterol. Modified Apo A-1 will tightly bind the artery wall, unlike the unmodified Apo A-1. Our study revealed different thing. In group with $\mathrm{HDL} \geq 40 \mathrm{mg} / \mathrm{dL}$, we found weak correlation between MPO activity with the Apo A-1 concentration in blood $(\mathrm{r}=0.250 ; \mathrm{p}=0.083)$. Besides, MPO activity also had very weak correlation with hs-CRP $(\mathrm{r}=0.140 ; \mathrm{p}=0.315)$, and unsurprisingly, the univariate or multivariate regression analysis of MPO did not show significant association with HDL type. MPO is not a strong indicator for the change of HDL characteristics from anti-inflammatory to proinflammatory. From this study, we found that SAA concentration in group with $\mathrm{HDL} \geq 40 \mathrm{mg} / \mathrm{dL}$ had weak correlation with Apo A-1 concentration in blood $(\mathrm{r}=0.261 ; \mathrm{p}=0.070)$, but had strong correlation with hsCRP $\left(r=0,453^{*} ; p=0.005\right)$. Based on previous reports by other researchers, the decrease in plasma Apo A-1 levels with induction of the acute phase response has been attributed to displacement of Apo A-1 from HDL particle with SAA in vivo and in in vitro (11). The real mechanism of the displacement of Apo A-1 with SAA, has not been known until now.

This study also showed that in the group with HDL $\geq 40 \mathrm{mg} / \mathrm{dL}$, the concentration of sPLA2 correlated weakly with the concentration of Apo A-1 in blood samples $\left(r=-0.303^{*} ; p=0.034\right)$. In the meantime, the concentration of sPLA2 had strong correlation with increased hs-CRP ( $\left.r=0.503^{*} ; \mathrm{p}=0.000\right)$. This finding was probably because we measured the concentration of the enzymes of sPLA2, and not directly the sPLA2 activity, and high concentration may not always mean high activity. The activity of sPLA2 in hydrolyzing phospholipids can release oxidized fatty acid from lipoprotein including HDL, which is pro-inflammatory and pro-atherogenic. The research by Rohrer showed a high concentration of CRP in elevated SPLA2 (12).

Relationship between HDL level and cardiovascular disease has been widely known. Low HDL level is related to high risk of CVD. In our study, $44.9 \%$ of individual with elevated HDL (considered protective) found to have high MPO, $51 \%$ had high sPLA2 and $57.14 \%$ had high SAA. On the whole, $87.8 \%$ of individual with elevated HDL level had high MPO and/or high sPLA2 and/or high SAA. Looking at the result of this study, it is appropriate if these markers are also observed at the time of performing other cardiovascular risk factors tests. The next thing is the estimation of the effect of MPO, sPLA2 and SAA on HDL function.

Association between SAA, sPLA2, MPO and the forming of pro-inflammatory HDL is very likely to be influenced by factor of time, how long the HDL is exposed with those three factors. In our study, SAA factor had better correlation with HDL than SPLA2 and MPO.

\section{Cause-Effect Relationship in Study Variables}

Interaction between the concentration of MPO, sPLA2 and SAA with pro-inflammatory HDL indicated that SAA was involved in HDL characteristics change from anti-inflammatory to pro-inflammatory, which was indicated with Apo A-1 level $<125 \mathrm{mg} / \mathrm{dL}$ and/or hs-CRP $\geq 1.1 \mathrm{mg} / \mathrm{L}$. This is suggested to be related to increasing in atherosclerosis risk. Thus, when early intervention can be done either by life style modification or therapeutic treatment which is intended to reduce this risk, the change of HDL characteristics from antiinflammatory to pro-inflammatory can indeed be 
inhibited and it can decrease the incidence of CVD in the future.

The exact mechanism of interaction among MPO, sPLA2 and SAA has not been known for certain.

The question need to be answered is how life style modification or early therapeutic treatment can reduce/decrease atherosclerosis progression? Related to that, our study result about SAA is expected not just to be able to estimate the change of HDL characteristics from anti-inflammatory to proinflammatory, but also to give additional information regarding the incidence of CVD. Therefore SAA determination, besides lipid profile and other risk factors of CVD, shows a promising future.

\section{Analysis of the Interaction of Variables in the Incidence of HDL Characteristics Change from anti-inflammatory to pro-inflammatory by pathobiological approach}

In analysing the incidence of HDL characteristics change from anti-inflammatory to proinflammatory, the core hypothesis approach of oxidative stress-inflammation-atherosclerosis is a powerful pathobiological mechanistic approach. The epidemiological and clinical facts provided convincing data about incidence of cardiovascular disease in individuals with normal HDL. In this study, we tried to observe the interaction of each variable in pathobiological concept in the incidence of HDL characteristics change from anti-inflammatory to pro-inflammatory.

In condition exposed with MPO, sPLA2 and SAA for a long time, HDL with anti-inflammatory characteristics undergo dysfunction and become proinflammatory. Pro-inflammatory characteristics of HDL are indicated by low Apo A-1 and/or elevated hs-CRP.

Our study resulted in a cut off value for SAA in HDL characteristics change from anti-inflammatory to pro-inflammatory. However, our study result was still unable to give explanation of how the mechanism of the anti-inflammatory HDL characteristics changed to anti-inflammatory, from a "good" microenvironment to a bad one. To explain the phenomena about the importance of "quality" besides "quantity", further deeper studies would be needed.

\section{Conclusions}

1. SAA concentration is independent and significantly associated with proinflammatory HDL type in healthy subjects.

2. SPLA2 concentration is not associated with proinflammatory HDL type in healthy subjects.

3. MPO concentration is not associated with proinflammatory HDL type in healthy subjects.

4. Interaction model among SAA, sPLA2 and MPO, showed that SAA has the best association with proinflammatory HDL type.

\section{Acknowledgement:}

The Authors thank to Endang Hoyaranda, Prodia Clinical Laboratory, Jakarta, and Gatot S. Lawrence, Vascular Research Unit Departement of Pathology, Wahidin Hospital, Makassar, for supporting this research.

\section{References:}

1. Honey K. Drug designed to raise HDL levels falls down. The Journal of Clinical Investigation. 2007; 117/2 : 282.

2. Navab M, Ananthramaiah GM, Reddy ST, et al. Thematic Review Series : The Pathogenesis of Atherosclerosis. The Oxidation Hypothesis of Atherogenesis: The Role of Oxidized Phospholipids and HDL. Journal of Lipid Research. 2004; 45: 993-1007.

3. Assmann G, Nofer JR. Atheroprotective effects of high-density lipoproteins. Ann Rev Med. 2004; 54 : 321-341.

4. Kontush A, Chantepie S, Chapman MJ. Small, dense HDL particles exert potent protection of atherogenic LDL against oxidative stress. Arterioscler Thromb Vasc Biol. 2003; 23: 1881-8.

5. Pirro M, Siepi D, Lupattelli G, et al. Plasma C-Reactive Protein in Subjects with Hypo/Hyperalphalipoproteinemias. Metabolism 2003, 52/4: 432-436

6. Van Lenten BJ, Hama SY, de Geer FC, et al. Anti-inflammatory HDL Becomes Proinflammatory during the Acute Phase Reaction. J. clin. invest 1995; 96: 2758-2767

7. Chait A, Han Cy, Oram JF, Heinecke JW. Lipoprotein. Associated inflammatory proteins: markers or mediators of cardiovascular disease? J. lipid Res 2005; 46: 389-403

8. Fogelman AM. When Good Cholesterol Goes Bad. Nature Medicine. 2004; 10/9: 902 - 903.

9. Zheng L, Nukuna B, Brennan M, et al. Apoliprotein A-1 is a selective target for myeloperoxidase, catalyzed oxidation and functional impairment in subjects with cardiovascular disease. The journal of Clinical Investigation 2004; 114/4: 529-541

10. Bergt C, Pennathur S, Fu X, et al. The myceoperoxidase product hypochlorous acid oxidizes HDL in the human artery wall and impairs ABC-A1 dependent cholesterol transport. PNAS 2004; 101/35: 13032-13037

11. Hosoai H, Webb NR, Glick JM, et al. Expression of serum Amyloid A protein in The Absence of The Acute Phase Response Does Not Reduce HDL-Cholesterol or Apo A-1 Levels in Human, Apo A-1 Transgenic Mice. J. lipid Res 1999; 40: 648-653

12). Rohrer L, Hersberger M, Eckardstein A. High Density Lipoproteins in The Intersection of Diabetes Mellitus, Inflammation and Cardiovascular Disease. Curr Opin Lipidol. 2004; 15: 269 -278.- 2756. 